\title{
GENERAL THEORY OF COUPLED THERMALLY STABLE ANISOTROPIC LAMINATES
}

\author{
PAOLO VANNUCCI
}

Preprint version

\begin{abstract}
A general theory of thermally stable anisotropic laminates with bending-extension coupling is presented in the framework of linear thermo-elasticity. It is based upon the use of tensor invariants, found by the polar formalism for bi-dimensional anisotropic elasticity. The extension of the results to moisture absorption effects is also proved.
\end{abstract}

KEywords: Thermal stability, Composite laminates, Tensor invariants, Polar method.

\section{INTRODUCTION}

A laminate having a coupling between the extension and the bending elastic response is said to be thermally stable when temperature variations, with respect to the manufacturing temperature $t_{0}$, do not produce geometrical variations of the plate, both for its dimensions and shape.

A complete theory of thermally stable laminates is proposed in this paper in the framework of the Classical Laminated Plates Theory (CLPT). It is well known that in this mechanical model, the fundamental kinematic assumption is the Kirchhoff's set of hypothesis: any straight line initally orthogonal to the mid-plane remains unstrained,

Date: July 17, 2012

Correspondence to: Paolo Vannucci

University of Versailles and Saint-Quentin-en-Yvelines

45, Avenue des Etats-Unis. 78035 Versailles (F)

paolo.vannucci@uvsq.fr . 
straight and perpendicular to the deformed mid-surface. The relevant consequence is that the displacement fields are linear across the thickness of the plate.

When thermal effects are of concern, some assumptions are to be added. In fact, in order to preserve, in the theory, the same type of kinematic assumptions, the laminate has to be subjected to linear through-the-thickness displacement fields caused by temperature variations. This can be obtained when (i) all the layers have the same thermal properties, as in the case of identical layers, and (ii) the temperature field is linear across the thickness, and this happens, if (i) is satisfied, when the heat flux is steady through the plate. For these reasons, the theory of this paper concerns laminates composed of identical layers and submitted to a field of temperature which is linear across the thickness of the plate. It is worth noting that the assumption of identical layers is also necessary for obtaining general solutions, i.e. valid for every material.

The problem of obtaining coupled thermally stable laminates has a certain importance in practical applications. Coupled laminates can be used, for instance, for obtaining a passive control of the twist of rotor blades of helicopters or of wind-mills. Usually, structural composite laminates are obtained curing pre-pregs layers, at temperatures that can be of the order of $180^{\circ} \mathrm{C}$ or more. Bending-extension uncoupling automatically ensures, for laminates made of identical layers, also thermal stability, see Section 2, but when coupled laminates are sought for, if no care is taken about the thermal stability of the plate, this last will immediately warp once the curing process is over, because of the gap between the curing temperature and the room temperature. So, whenever coupled laminates are to be manufactured, they have to be designed thermally stable, in order to avoid the loss of geometry of the plate.

Very few works exist on this topic and none of them considers the problem of thermal stability in a general way: normally, only sufficient conditions for obtaining warp-free laminates are considered. The first studies on the subject, actually papers on the phenomenon of thermal warping, seem to be appeared in 1981, [6], [5], [3]. Winckler, in his $\mathrm{PhD}$ thesis and in some related papers, [19], [20], has been, perhaps, the first to propose some sufficient rules for the design of warp-free coupled laminates. Chen has then given some other sufficient rules on the subject, [1], as well as Cross et al, [2], in a paper presenting some families of warp-free laminates, work extended in a subsequent paper by Haynes and Armanios, [4]. In these last two papers, the authors also look for stacking sequences optimizing the thermal warping. Nevertheless, none of these papers presents a general theory of anisotropic coupled thermally stable laminates: this paper tries to give an answer to this question. The results are valid also for the effects produced by moisture absorption, as shown in the paper.

\section{Recall of the fundamental EQUations}

Any linear thermal field characterized by a temperature $t_{u p}$ and $t_{b o t}$, respectively on the upper and lower surfaces of a plate, can be decomposed in the sum of two different fields: 
- a constant field of value

$$
t=\frac{t_{u p}+t_{b o t}}{2}-t_{0}
$$

- an antisymmetric linear field characterised by the constant gradient

$$
\nabla t=\frac{t_{u p}-t_{b o t}}{h}
$$

with $h$ the total thickness of the laminate.

The decomposition of the thermal field in the two parts above is rather classical, but in the case of coupled laminates it assumes an increased importance. In fact, in classical plates and in uncoupled laminates too, the field $t$ produces in-plane strains of the midplane only, i.e. contraction or expansion, but not out-of-plane deformations, while $\nabla t$ does not change the mid-plane dimensions, but makes the plate bend and warp. These effects are well known and in a sense natural, because originated by the fact that the material has some given coefficients of thermal expansion, that in general are not identical in all the directions for anisotropic plates. The key point in the present research is that the above phenomenon does not happen in the same way in bending-extension coupled laminates.

In fact, let us consider the constitutive law of laminates obtained in the framework of the CLPT, cfr., e.g., [7], when the plate is submitted to in-plane actions and bending moments produced not only by applied forces but also by thermal fields:

$$
\left\{\begin{array}{l}
\mathbf{N} \\
\mathbf{M}
\end{array}\right\}=\left[\begin{array}{ll}
\mathbf{A} & \mathbf{B} \\
\mathbf{B} & \mathbf{D}
\end{array}\right]\left\{\begin{array}{c}
\varepsilon \\
\kappa
\end{array}\right\}+t\left\{\begin{array}{l}
\mathbf{U} \\
\mathbf{V}
\end{array}\right\}+\nabla t\left\{\begin{array}{c}
\mathbf{V} \\
\mathbf{W}
\end{array}\right\} .
$$

In the above equation, $\mathbf{N}$ and $\mathbf{M}$ are the tensor fields of in-plane internal actions, i.e. of in-plane forces and bending moments respectively, $\varepsilon$ is the in-plane strain tensor and $\kappa$ the tensor of curvatures of the mid-plane. The link between the internal actions and deformation tensors is provided by three fourth-rank tensors, $\mathbf{A}, \mathbf{B}$ and $\mathbf{D}$, while three second-rank tensors, $\mathbf{U}, \mathbf{V}$ and $\mathbf{W}$ relate the internal actions to the thermal field. Tensors $\mathbf{A}$ and $\mathbf{D}$ describe respectively the extension and bending elastic response of the laminate, and they have all the classical properties of an elasticity tensor: minor and major tensor symmetries, positive definiteness. Tensor $\mathbf{B}$ is the bending-extension coupling tensor; it is not a true elastic tensor, because it not positive definite, actually it is not definite. This stiffness coupling tensor has nevertheless both the major and minor tensor symmetries. It is apparent from eq. (3) that $\mathbf{B}$ couples in-plane internal forces with curvatures and, at the same time, bending moments with in-plane strains. Of course, a laminate is said bending-extension uncoupled when

$$
\mathbf{B}=\mathbf{O} .
$$


Tensors $\mathbf{A}, \mathbf{B}$ and $\mathbf{D}$ are given, for laminates composed of identical layers, by the following homogenization laws:

$$
\begin{aligned}
& \mathbf{A}=\frac{h}{n} \sum_{j=1}^{n} \mathbf{Q}\left(\delta_{j}\right), \\
& \mathbf{B}=\frac{1}{2}\left(\frac{h}{n}\right)^{2} \sum_{j=1}^{n} b_{j} \mathbf{Q}\left(\delta_{j}\right), \\
& \mathbf{D}=\frac{1}{12}\left(\frac{h}{n}\right)^{3} \sum_{j=1}^{n} d_{j} \mathbf{Q}\left(\delta_{j}\right),
\end{aligned}
$$

where

$$
\begin{gathered}
b_{j}=2 j-n-1, \\
d_{j}=12 j(j-n-1)+4+3 n(n+2),
\end{gathered}
$$

with $n$ the number of layers and $\mathbf{Q}\left(\delta_{j}\right)$ the reduced stiffness tensor of the basic layer $j$, oriented at the angle $\delta_{j}$.

The second rank symmetric tensors $\mathbf{U}, \mathbf{V}$ and $\mathbf{W}$ plays respectively the same role of $\mathbf{A}, \mathbf{B}$ and $\mathbf{D}$, but for the part of the internal actions produced by the thermal field. Namely, $\mathbf{U}$ is the tensor of in-plane actions per unit of temperature $t$ and $\mathbf{W}$ the tensor of bending moments per unit of thermal gradient $\nabla t$. $\mathbf{V}$ is a coupling tensor that describes at the same time the in-plane actions produced by a unitary $\nabla t$ and the bending moments produced by a unit field $t$. Unlike the case of $\mathbf{B}$, we will see in the following that defining a thermo-elastically uncoupled laminate is not trivial: the condition $\mathbf{V}=\mathbf{O}$ is not, generally speaking, sufficient to ensure a complete thermo-elastic uncoupling. The expression of $\mathbf{U}, \mathbf{V}$ and $\mathbf{W}$ is quite similar to that of $\mathbf{A}, \mathbf{B}$ and $\mathbf{D}$, in particular coefficients $b_{j}$ and $d_{j}$ are still given by eqs. (6):

$$
\begin{aligned}
& \mathbf{U}=\frac{h}{n} \sum_{j=1}^{n} \gamma\left(\delta_{j}\right), \\
& \mathbf{V}=\frac{1}{2}\left(\frac{h}{n}\right)^{2} \sum_{j=1}^{n} b_{j} \gamma\left(\delta_{j}\right), \\
& \mathbf{W}=\frac{1}{12}\left(\frac{h}{n}\right)^{3} \sum_{j=1}^{n} d_{j} \gamma\left(\delta_{j}\right),
\end{aligned}
$$

where the second-rank tensor $\gamma\left(\delta_{j}\right)$ is given by

$$
\gamma\left(\delta_{j}\right)=\mathbf{Q}\left(\delta_{j}\right) \alpha\left(\delta_{j}\right),
$$

and $\alpha\left(\delta_{j}\right)$ is the second-order tensor of thermal expansion coefficients of the basic layer, oriented at the angle $\delta_{j}$.

Composite materials with organic matrices are subjected also to another occurrence, the moisture absorption. This phenomenon happens, usually, with a different time scale with respect to thermal changes, so these two occurrences are normally unrelated. Nevertheless, moisture absorption is ruled by equations that are completely similar to those concerning thermal effects. In particular, in the presence of a linear variation of the moisture concentration $c$ across the thickness, the constitutive law for a laminate is 
similar to that of eq. (3),

$$
\left\{\begin{array}{l}
\mathbf{N} \\
\mathbf{M}
\end{array}\right\}=\left[\begin{array}{cc}
\mathbf{A} & \mathbf{B} \\
\mathbf{B} & \mathbf{D}
\end{array}\right]\left\{\begin{array}{l}
\boldsymbol{\varepsilon} \\
\boldsymbol{\kappa}
\end{array}\right\}+c\left\{\begin{array}{l}
\mathbf{F} \\
\mathbf{G}
\end{array}\right\}+\nabla c\left\{\begin{array}{l}
\mathbf{G} \\
\mathbf{H}
\end{array}\right\},
$$

where tensors $\mathbf{A}, \mathbf{B}$ and $\mathbf{D}$ are exactly the same defined above, eq. (5), while the second rank tensors $\mathbf{F}, \mathbf{G}$ and $\mathbf{H}$ are the corresponding, for the elastic response to moisture absorption, of tensors $\mathbf{U}, \mathbf{V}$ and $\mathbf{W}$ respectively. In particular, eqs. (7) give also $\mathbf{F}, \mathbf{G}$ and $\mathbf{H}$ if tensor $\gamma$ is replaced by the second-rank tensor

$$
\lambda\left(\delta_{j}\right)=\mathbf{Q}\left(\delta_{j}\right) \beta\left(\delta_{j}\right),
$$

where $\beta\left(\delta_{j}\right)$ is the second-order tensor of moisture expansion coefficients of the basic layer, oriented at the angle $\delta_{j}$. Formally, the phenomenon of moisture absorption in a laminated plate is hence ruled by equations that are algebraically completely identical to those that govern the phenomenon of thermal expansion. Then, if tensors $\alpha$ and $\beta$ have the same type of orthotropy, all the results that can be found for a case are valid also for the other one.

This assumption will be done in the following; it is physically motivated because the orthotropy of composite layers is induced by the presence, in an isotropic matrix, of oriented fibres. This hypothesis corresponds hence to the physical fact that the fibres produce the same type of anisotropy for the thermal and moisture expansion coefficients, which seems to be reasonable. Hence, all the results found for the thermal case are qualitatively the same also for the moisture absorption case; that is why in the following of the paper only the thermal case will be considered, never forgetting that all that will be said can be repeated verbatim for the effects of moisture absorption.

\section{Thermal StaBility}

The thermal effects on a coupled plate can be better analysed introducing the inverse of eq. (3):

$$
\left\{\begin{array}{c}
\varepsilon \\
\boldsymbol{\kappa}
\end{array}\right\}=\left[\begin{array}{cc}
\mathbf{a} & \mathbf{b} \\
\mathbf{b}^{\mathrm{T}} & \mathbf{d}
\end{array}\right]\left\{\begin{array}{c}
\mathbf{N} \\
\mathbf{M}
\end{array}\right\}+t\left\{\begin{array}{c}
\mathbf{u} \\
\mathbf{v}_{\mathbf{2}}
\end{array}\right\}+\nabla t\left\{\begin{array}{l}
\mathbf{v}_{\mathbf{1}} \\
\mathbf{w}
\end{array}\right\} .
$$

In eq. (11), tensors $\mathbf{a}, \mathbf{b}$ and $\mathbf{d}$ are the compliance tensors corresponding to the stiffness tensors $\mathbf{A}, \mathbf{B}$ and $\mathbf{D}$ respectively. To remark that the compliance coupling tensor has not the major tensor symmetries: $b_{p q r s} \neq b_{r s p q}$. Nevertheless, it has still six independent components. Tensors $\mathbf{u}, \mathbf{v}_{1}, \mathbf{v}_{2}$ and $\mathbf{w}$ are the compliance corresponding of tensors $\mathbf{U}$, $\mathbf{V}$ and $\mathbf{W}$. The compliance tensors can be given as functions of the stiffness tensors, [16]:

$$
\begin{gathered}
\mathbf{a}=\left(\mathbf{A}-\mathbf{B D}^{-1} \mathbf{B}\right)^{-1}, \\
\mathbf{b}=\left\{\begin{array}{l}
-\mathbf{a} \mathbf{B} \mathbf{D}^{-1}=-\left(\mathbf{A}-\mathbf{B D}^{-1} \mathbf{B}\right)^{-1} \mathbf{B} \mathbf{D}^{-1} \\
-\mathbf{A}^{-1} \mathbf{B} \mathbf{d}=-\mathbf{A}^{-1} \mathbf{B}\left(\mathbf{D}-\mathbf{B} \mathbf{A}^{-1} \mathbf{B}\right)^{-1}
\end{array}\right.
\end{gathered}
$$




$$
\begin{aligned}
& \mathbf{b}^{\mathrm{T}}=\left\{\begin{array}{l}
-\mathbf{d B A}^{-1}=-\left(\mathbf{D}-\mathbf{B} \mathbf{A}^{-1} \mathbf{B}\right)^{-1} \mathbf{B} \mathbf{A}^{-1}, \\
-\mathbf{D}^{-1} \mathbf{B} \mathbf{a}=-\mathbf{D}^{-1} \mathbf{B}\left(\mathbf{A}-\mathbf{B D}^{-1} \mathbf{B}\right)^{-1},
\end{array}\right. \\
& \mathbf{d}=\left(\mathbf{D}-\mathbf{B} \mathbf{A}^{-1} \mathbf{B}\right)^{-1}, \\
& \mathbf{u}=\mathbf{a} \mathbf{U}+\mathbf{b} \mathbf{V}=\mathbf{a}\left(\mathbf{U}-\mathbf{B D}^{-1} \mathbf{V}\right)=\left(\mathbf{A}-\mathbf{B D}^{-1} \mathbf{B}\right)^{-1}\left(\mathbf{U}-\mathbf{B D}^{-1} \mathbf{V}\right), \\
& \mathbf{v}_{1}=\mathbf{a} \mathbf{V}+\mathbf{b} \mathbf{W}=\mathbf{a}\left(\mathbf{V}-\mathbf{B D}^{-1} \mathbf{W}\right)=\left(\mathbf{A}-\mathbf{B D}^{-1} \mathbf{B}\right)^{-1}\left(\mathbf{V}-\mathbf{B D}^{-1} \mathbf{W}\right), \\
& \mathbf{v}_{2}=\mathbf{b}^{\mathrm{T}} \mathbf{U}+\mathbf{d} \mathbf{V}=\mathbf{d}\left(\mathbf{V}-\mathbf{B A}^{-1} \mathbf{U}\right)=\left(\mathbf{D}-\mathbf{B A}^{-1} \mathbf{B}\right)^{-1}\left(\mathbf{V}-\mathbf{B A}^{-1} \mathbf{U}\right), \\
& \mathbf{w}=\mathbf{b}^{\mathrm{T}} \mathbf{V}+\mathbf{d} \mathbf{W}=\mathbf{d}\left(\mathbf{W}-\mathbf{B A}^{-1} \mathbf{V}\right)=\left(\mathbf{D}-\mathbf{B A}^{-1} \mathbf{B}\right)^{-1}\left(\mathbf{W}-\mathbf{B} \mathbf{A}^{-1} \mathbf{V}\right) .
\end{aligned}
$$

There are two thermo-elastic compliance coupling tensors $\mathbf{v}_{1}$ and $\mathbf{v}_{2}$, that are distinct but not independent:

$$
\mathbf{v}_{1}=\mathbf{a}\left[\mathbf{d}^{-1} \mathbf{v}_{2}+\mathbf{B}\left(\mathbf{A}^{-1} \mathbf{U}-\mathbf{D}^{-1} \mathbf{W}\right)\right] .
$$

We are interested here in what happens when the coupled laminate is subjected to only thermal fields, i.e. when $\mathbf{N}$ and $\mathbf{M}$ are null. Eq. (11) clearly shows that a thermal field $t$, as said above, produce a planar strain of the mid-plane, described by tensor $\mathbf{u}$, which is the tensor of thermal expansion coefficients of the plate, but if the laminate is thermally coupled, also a bending of the plate described by the thermal coupling tensor $\mathbf{v}_{2}$. This coupling tensor $\mathbf{v}_{2}$ is responsible of the warping of thermally coupled laminates produced by a uniform thermal field $t$, as it happens after curing.

In addition, still considering eq. (11), it is clear that a gradient thermal field $\nabla t$ will bend the plate, which is described by the tensor of thermal curvature coefficients $\mathbf{w}$, but if the plate is thermally coupled, then this gradient field will produce also a strain of the mid-plane, described by the thermal coupling tensor $\mathbf{v}_{1}$.

A general and complete definition of thermal stability is used in this paper: a laminate is said to be thermally stable when

$$
\left\{\begin{array}{l}
\mathbf{v}_{1}=\mathbf{O} \\
\mathbf{v}_{2}=\mathbf{O} .
\end{array}\right.
$$

Nevertheless, the effect induced by tensor $\mathbf{v}_{2}$ is by far much more important. This because such effect is permanently present in non thermally-stable laminates obtained by a curing process, and in this case $t$ can be very high (up to about $250^{\circ} \mathrm{C}$ ). In addition, as laminates are often thin plates, the thermal warping produced by a field $t$ can be very important, i.e. it can produce dramatic geometrical and mechanical effects (namely, great displacements and considerable decrease of the buckling loads). That is why this is the unique effect normally considered by designers, and it normally denotes the thermal stability in the papers on the subject. The object of this paper being a complete and general analysis of thermal-stability, the above condition (21) is the necessary starting point. 
In this paper, thermal stability will be analyzed at first for what concerns tensors $\mathbf{v}_{1}$ and $\mathbf{v}_{2}$ separately; then, the conditions for satisfying eqs. (21) at the same time will be also introduced. Finally, the complete absence of thermal effects on coupled laminates will be considered too. Except for the case of tensor $\mathbf{v}_{2}$, all these points are new and not considered in the papers cited in Section 1.

\section{INTRODUCING THE POLAR FORMALISM}

The composition laws of eqs. (5) and (7) do not depend on the tensor representation. So, they are written below using the polar formalism for plane tensors introduced by Verchery, [17], that for laminates composed by identical layers gives:

$$
\left\{\begin{array}{l}
T_{0}^{A}=h T_{0}, \\
T_{1}^{A}=h T_{1}, \\
R_{0}^{A} e^{4 i \Phi_{0}^{A}}=h R_{0} e^{4 i \Phi_{0}}\left(\zeta_{0}+i \zeta_{2}\right), \\
R_{1}^{A} e^{2 i \Phi_{1}^{A}=h R_{1} e^{2 i \Phi_{1}}\left(\zeta_{1}+i \zeta_{3}\right)} ;
\end{array}\right.
$$

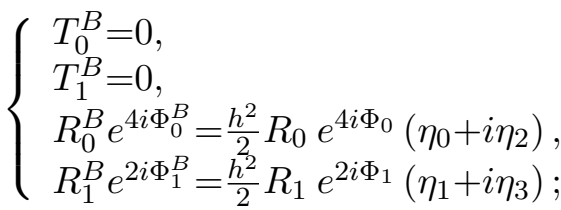

$$
\left\{\begin{array}{l}
T_{0}^{D}=\frac{h^{3}}{12} T_{0}, \\
T_{1}^{D}=\frac{h^{3}}{12} T_{1}, \\
R_{0}^{D} e^{4 i \Phi_{0}^{D}}=\frac{h^{3}}{12} R_{0} e^{4 i \Phi_{0}}\left(\xi_{0}+i \xi_{2}\right), \\
R_{1}^{D} e^{2 i \Phi_{1}^{D}}=\frac{h^{3}}{12} R_{1} e^{2 i \Phi_{1}}\left(\xi_{1}+i \xi_{3}\right)
\end{array}\right.
$$

$$
\left\{\begin{array}{l}
T^{U}=h T^{\gamma} \\
R^{U} e^{2 i \Phi^{U}}=h R^{\gamma} e^{2 i \Phi^{\gamma}}\left(\zeta_{1}+i \zeta_{3}\right)
\end{array}\right.
$$

$$
\left\{\begin{array}{l}
T^{V}=0, \\
R^{V} e^{2 i \Phi^{V}}=\frac{h^{2}}{2} R^{\gamma} e^{2 i \Phi^{\gamma}}\left(\eta_{1}+i \eta_{3}\right)
\end{array}\right.
$$

$$
\left\{\begin{array}{l}
T^{W}=\frac{h^{3}}{12} T^{\gamma} \\
R^{W} e^{2 i \Phi^{W}}=\frac{h^{3}}{12} R^{\gamma} e^{2 i \Phi^{\gamma}}\left(\xi_{1}+i \xi_{3}\right)
\end{array}\right.
$$


In the above equations, $\zeta_{0}, \eta_{0}, \xi_{0}$ etc. are the so-called lamination parameters, introduced by Tsai and Hahn, [8]:

$$
\begin{aligned}
& \left(\zeta_{0}+i \zeta_{2}\right)=\frac{1}{n} \sum_{j=1}^{n} e^{4 i \delta_{j}}, \\
& \left(\zeta_{1}+i \zeta_{3}\right)=\frac{1}{n} \sum_{j=1}^{n} e^{2 i \delta_{j}}, \\
& \left(\eta_{0}+i \eta_{2}\right)=\frac{1}{n^{2}} \sum_{j=1}^{n} b_{j} e^{4 i \delta_{j}}, \\
& \left(\eta_{1}+i \eta_{3}\right)=\frac{1}{n^{2}} \sum_{j=1}^{n} b_{j} e^{2 i \delta_{j}}, \\
& \left(\xi_{0}+i \xi_{2}\right)=\frac{1}{n^{3}} \sum_{j=1}^{n} d_{j} e^{4 i \delta_{j}}, \\
& \left(\xi_{1}+i \xi_{3}\right)=\frac{1}{n^{3}} \sum_{j=1}^{n} d_{j} e^{2 i \delta_{j}} .
\end{aligned}
$$

For a laminate composed of identical layers, lamination parameters can be used to represent the whole influence of the geometry of the stacking sequence on the behaviour of the plate. Eqs. (22) - (27) clearly show that the influence of the geometry of the stack and that of the material properties have been completely separated: the first one is represented by the lamination parameters, while the second one by the polar parameters of the material.

Conventionally, a polar component without superscript indicates a quantity proper to the basic layer, i.e. to $\mathbf{Q}$ or to $\alpha$, as in the right sides of eqs. (22) - (24). In eqs. (25) - (27), $T^{\gamma}, R^{\gamma}$ and $\Phi^{\gamma}$ are the polar components of tensor $\gamma$, eq. (8). In the following, index $L$ will indicate, when needed, the polar parameters of a generic tensor $\mathbf{L}$ among $\mathbf{A}, \mathbf{B}, \mathbf{D}, \mathbf{U}$ etc.

The Cartesian components of $\mathbf{A}, \mathbf{B}, \mathbf{D}$, a or $\mathbf{d}$ can be given as functions of its polar parameters. For instance, for $\mathbf{A}$ it is:

$$
\left\{\begin{array}{l}
A_{x x x x}(\theta)=T_{0}^{A}+2 T_{1}^{A}+R_{0}^{A} \cos 4\left(\Phi_{0}^{A}-\theta\right)+4 R_{1}^{A} \cos 2\left(\Phi_{1}^{A}-\theta\right), \\
A_{x x x y}(\theta)=R_{0}^{A} \sin 4\left(\Phi_{0}^{A}-\theta\right)+2 R_{1}^{A} \sin 2\left(\Phi_{1}^{A}-\theta\right), \\
A_{x x y y}(\theta)=-T_{0}^{A}+2 T_{1}^{A}-R_{0}^{A} \cos 4\left(\Phi_{0}^{A}-\theta\right), \\
A_{x y x y}(\theta)=T_{0}^{A}-R_{0}^{A} \cos 4\left(\Phi_{0}^{A}-\theta\right) \\
A_{x y y y}(\theta)=-R_{0}^{A} \sin 4\left(\Phi_{0}^{A}-\theta\right)+2 R_{1}^{A} \sin 2\left(\Phi_{1}^{A}-\theta\right), \\
A_{y y y y}(\theta)=T_{0}^{A}+2 T_{1}^{A}+R_{0}^{A} \cos 4\left(\Phi_{0}^{A}-\theta\right)-4 R_{1}^{A} \cos 2\left(\Phi_{1}^{A}-\theta\right),
\end{array}\right.
$$

and similar equations hold of course for $\mathbf{B}, \mathbf{D}$, $\mathbf{a}$ and $\mathbf{d}$, but not for $\mathbf{b}$, because it does not have the major symmetries. In eq. (29), the Cartesian components are those in a frame rotated counter clockwise through an angle $\theta$ with respect to a fixed frame that can be chosen fixing one of the two polar angles; the usual choice is $\Phi_{1}=0$.

The Cartesian components of $\mathbf{U}$ are

$$
\left\{\begin{array}{l}
U_{x x}(\theta)=T^{U}+R^{U} \cos 2\left(\Phi^{U}-\theta\right) \\
U_{x y}(\theta)=R^{U} \sin 2\left(\Phi^{U}-\theta\right) \\
U_{y y}(\theta)=T^{U}-R^{U} \cos 2\left(\Phi^{U}-\theta\right)
\end{array}\right.
$$


and similar relations hold for $\mathbf{V}, \mathbf{W}, \mathbf{u}, \mathbf{v}_{1}, \mathbf{v}_{2}$ and $\mathbf{w}$. Also in this case, choosing a value for $\Phi^{U}$ means to fix a frame. To remark that unlike tensor $\mathbf{b}$, which has not the major tensor symmetries, tensors $\mathbf{v}_{1}$ and $\mathbf{v}_{2}$ are symmetric, as it can be easily proven by eqs. (17) and (18), thanks to the minor tensor symmetries of $\mathbf{b}$.

As already said, in eqs. (29) and (30) $T_{0}, T_{1}, R_{0}, R_{1}, T$ and $R$ are the polar moduli of the basic layer, while $\Phi_{0}, \Phi_{1}$ and $\Phi$ are the polar angles. All the polar moduli and the angular difference $\Phi_{0}-\Phi_{1}$ corresponding to a given tensor, constitute a complete set of independent invariants for that tensor.

Inserting eq. (29) into eq. (22) allows for expressing the Cartesian components of $\mathbf{A}$ as functions of the polar parameters of the basic layer; of course, similar expressions can be obtained for all the other stiffness tensors.

The use of the polar formalism is useful for several reasons. First of all, equations like eqs. (29) and (30) show immediately that in the polar method, the isotropic part, represented by invariants $T_{0}^{L}, T_{1}^{L}$ and $T^{L}$, is separated form the anisotropic one, represented by invariants $R_{0}^{L}, R_{1}^{L}, \Phi_{0}^{L}-\Phi_{1}^{L}$ and $R^{L}$.

In addition, equations like (22) and (25), show that the isotropic part is completely determined by the thickness of the laminate and by the choice of the material composing the basic layer, i.e., in the case of laminates composed of identical layers, it cannot be modified by the design process. So, when the layers are identical, the design process can act only upon the anisotropic part of each tensor. Equations (23) and (26) show in addition that in such cases, the coupling stiffness tensors $\mathbf{B}$ and $\mathbf{V}$ have a null isotropic part. In a sense, uncoupling corresponds to annihilate their anisotropic part or, which is the same, to impose that they are isotropic. An accessory result is that, being null the isotropic part of $\mathbf{V}$, it is $V_{x x}(\theta)=-V_{y y}(\theta) \forall \theta$, a result already given in [2] but only for the case $\theta=0$.

The polar formalism gives immediately another result, that is of some importance in the following: comparing eqs. (23) and (26) shows that the sum appearing in the anisotropic part of $\mathbf{V}$ is just the same of the second anisotropic polar component of $\mathbf{B}$, eq. $\left(23_{4}\right)$. Then, taking into account this and eqs. (12) to (19), bending-extension uncoupling gives

$$
\mathbf{B}=\mathbf{O} \Rightarrow\left\{\begin{array}{l}
\mathbf{b}=\mathbf{V}=\mathbf{v}_{1}=\mathbf{v}_{2}=\mathbf{O} \\
\mathbf{a}=\mathbf{A}^{-1} \\
\mathbf{d}=\mathbf{D}^{-1} \\
\mathbf{u}=\mathbf{A}^{-1} \mathbf{U} \\
\mathbf{w}=\mathbf{D}^{-1} \mathbf{W}
\end{array}\right.
$$

So, the bending-extension uncoupling is, for laminates composed of identical layers, a very strong condition: it annihilates all the other couplings. Nevertheless, it is evident that, generally speaking, the converse is not true: $\mathbf{B}$ has an anisotropic polar component, $\left(23_{3}\right)$, that is not necessarily null when $\mathbf{V}=\mathbf{O}$, eq. (26). Also, eq. (13) shows that it is impossible to have $\mathbf{b}=\mathbf{O}$ when $\mathbf{B} \neq \mathbf{O}$, because this should necessarily imply

$$
\mathbf{A B}^{-1}=\mathbf{B D}^{-1}
$$


putting this condition into eqs. (12) or (15) gives the null tensor for a or $\mathbf{d}$, which is mechanically impossible: $\mathbf{a}$ and $\mathbf{d}$ are compliance tensors, they are positive definite.

In addition, as we will see, the different thermo-elastic coupling tensors $\mathbf{V}, \mathbf{v}_{1}$ and $\mathbf{v}_{2}$ can be null independently from each other and from $\mathbf{B}$ (this result is not true for hybrid laminates, i.e. composed of layers that are different). This fact renders the analysis of thermal stability rather cumbersome.

Another advantage of the polar method, is its power in intrinsically describing anisotropy. In fact, cfr. [12], ordinary orthotropy is determined by the invariant condition

$$
\Phi_{0}^{L}-\Phi_{1}^{L}=K^{L} \frac{\pi}{4}, \quad K^{L} \in\{0,1\},
$$

while two types of special orthotropies exist for stiffness: square symmetry, i.e. the planar corresponding of cubic syngony, characterized by the invariant condition

$$
R_{1}^{L}=0,
$$

and the so-called $R_{0}$ - orthotropy, [9] determined by the invariant condition

$$
R_{0}^{L}=0 .
$$

To be remarked that the term special orthotropy is used here in a sense which is different from that, rather misleading, normally understood by people working with laminates, as for instance in [2]. In fact, usually the expression specially orthotropic indicates that an orthotropic ply has the orthotropy axes parallel to the reference frame. Hence, the term special is used only to recall that the stiffness matrix is particularly simple in such a situation, and not that the material can belong to a special type of orthotropy, like those determined by conditions (34) and (35). In this paper, the expression special orthotropy has a precise and invariant mathematical and mechanical meaning: it indicates a tensor characterized by one of the two conditions (34) or (35), but not by eq. (33). From a mathematical point of view, the tensor invariant in eq. (33) is of a higher order of those in eqs. (34) and (35), [15], while mechanically, the three cases of orthotropy are characterized by different behaviours and consequences, see below or also [12] and [13].

The most general expression for the determinant of $\mathbf{B}$, in the case of identical layers, can be easily computed introducing eq. (23) into the corresponding of eq. (29) and then calculating the determinant of the resulting matrix representing $\mathbf{B}$; the result is

$$
\operatorname{det} \mathbf{B}=16 R_{0}^{B}\left(R_{1}^{B}\right)^{2} \cos 4\left(\Phi_{0}^{B}-\Phi_{1}^{B}\right) .
$$

This result shows immediately, through conditions (34) and (35) applied to tensor $\mathbf{B}$, that $\mathbf{B}$ is singular whenever it has a special orthotropy. The same equation shows also that det $\mathbf{B}$ can take any positive, negative or also null value: $\mathbf{B}$ is not definite, as already said.

The role played by the type of orthotropy, i.e. by parameters $R_{0}^{L}$ and $R_{1}^{L}$ on the design of laminates is widely discussed in [12]; their influence on thermal stability solutions will be considered in the following sections. 


\section{WARP-FREE COUPLED LAMINATES}

For the sake of conciseness, a warp-free laminate will indicate in the following a laminate that does not warp when only the thermal field $t$ is present. The conditions giving warpfree laminates are considered in this section; the objective is to obtain coupled laminates satisfying eq. (212). Eq. (18) gives two different cases for having $\mathbf{v}_{2}=\mathbf{O}$ when $\mathbf{B} \neq \mathbf{O}$, they are examined below, separately.

5.1. First type of coupled warp-free laminates. The first type of warp-free coupled laminates is composed of all the plates that satisfy the following conditions:

$$
\left\{\begin{array}{l}
\mathbf{B} \neq \mathbf{O} \\
\mathbf{V}=\mathbf{O} \\
\mathbf{b}^{\mathrm{T}} \mathbf{U}=\mathbf{O}
\end{array}\right.
$$

Conditions (37) are clearly sufficient conditions to obtain a coupled laminate that satisfies eq. (18), i.e. a warp-free coupled laminate. Some remarks are to be done concerning this case, they are listed below.

Remark R1. It is possible to have laminates with $\mathbf{V}=\mathbf{O}$ but $\mathbf{v}_{2} \neq \mathbf{O}$; it is sufficient that eq. $\left(37_{3}\right)$ is not satisfied.

Remark R2. Being $\mathbf{V}=\mathbf{O}$ and the coefficients of thermal expansion not isotropic, i.e. $R \neq$ 0 , eq. $\left(26_{2}\right)$ implies that $R_{1}^{B}=0$, eq. $\left(23_{4}\right)$. So, $\mathbf{B}$ is necessarily square symmetric:

$$
\mathbf{B}=R_{0}^{B}\left[\begin{array}{rrr}
\cos 4 \Phi_{0}^{B} & -\cos 4 \Phi_{0}^{B} & \sin 4 \Phi_{0}^{B} \\
-\cos 4 \Phi_{0}^{B} & \cos 4 \Phi_{0}^{B} & -\sin 4 \Phi_{0}^{B} \\
\sin 4 \Phi_{0}^{B} & -\sin 4 \Phi_{0}^{B} & -\cos 4 \Phi_{0}^{B}
\end{array}\right] .
$$

Hence, $\mathbf{B}$ is defined by only one invariant, $R_{0}^{B}$.

Remark R3. As $\mathbf{B}$ has a special orthotropy, it is singular: $\operatorname{det} \mathbf{B}=0$, as said in section 4 , what is easy to be verified through eq. (38). Hence, $\mathbf{B}$ is singular whenever the thermo-elastic coupling stiffness tensor $\mathbf{V}$ is null.

Remark R4. As tensor $\mathbf{U}$ is never the null tensor, condition (373) can be satisfied only if

$$
\operatorname{det} \mathbf{b}=0 \text {. }
$$

Using the Binet's theorem in eq. (13) gives

$$
\operatorname{det} \mathbf{b}=\operatorname{det}\left(-\mathbf{a} \mathbf{B} \mathbf{D}^{-1}\right)=-\frac{\operatorname{det} \mathbf{a} \operatorname{det} \mathbf{B}}{\operatorname{det} \mathbf{D}},
$$

and considering that in this case it is $\operatorname{det} \mathbf{B}=0$, gives immediately that condition (39) is satisfied. Hence, the special orthotropy of $\mathbf{B}$ is a sufficient condition for satisfying eq. (39) too. 
Remark R5. Condition $\left(37_{3}\right)$ can be interpreted as an eigenvector problem: $\mathbf{U}$ has to be the eigentensor of $\mathbf{b}^{\mathrm{T}}$ corresponding to the null eigenvalue. Nevertheless, one should remember that in a design problem all the tensors are related, because changing the orientations of the layers changes all the tensors describing the elastic and thermo-elastic behaviour of the plate. Though it is too involved to explicit all the links between the different tensors, these links exist: in eq. $\left(37_{3}\right), \mathbf{b}$ and $\mathbf{U}$ are not independent. So, the design problem of this first type of warp-free coupled laminates corresponds to look for laminates respecting conditions (37): what said above shows that these conditions are compatible, so these laminates can exist and resuming they have

$$
\begin{aligned}
& \mathbf{B} \neq \mathbf{O}, \quad \mathbf{b} \neq \mathbf{O}, \quad \mathbf{V}=\mathbf{O}, \quad \mathbf{v}_{2}=\mathbf{O}, \\
& \operatorname{det} \mathbf{B}=0, \quad \operatorname{det} \mathbf{b}=0, \quad R_{1}^{B}=0 .
\end{aligned}
$$

Remark R6. It is not possible to give general solutions for the design of laminates of this first type. Nevertheless, it is possible to give some sufficient conditions for obtaining such laminates. The first one is rather simple: laminates composed of square symmetric layers automatically satisfy conditions (37). This is the case of layers reinforced by balanced fabrics, i.e. having the same amount of fibres in warp and weft. In fact, in such cases the condition of square symmetry of the ply, $R=R_{1}=0$, gives, through equations (22) $-(27)$, that it is also

$$
R=R_{1}=0 \quad \Rightarrow \quad R_{1}^{A}=R_{1}^{B}=R_{1}^{D}=R^{U}=R^{V}=R^{W}=0 .
$$

Then, conditions $\left(37_{2}\right)$ and (39) are satisfied; it can be verified that also condition $\left(37_{3}\right)$ is always satisfied in this case, this has been done using a code for the formal development of equations; unfortunately, the analytical developments are very long and it is not possible to show them here in detail. So, laminates composed by identical layers reinforced by balanced fabrics are always warp free; using a stacking sequence which is not uncoupled, gives finally a laminate that satisfy all the conditions (37).

Remark R7. Another, less restrictive, sufficient condition is the following one:

$$
R_{1}^{A}=R_{1}^{B}=0 .
$$

This is the case of laminates that have $\mathbf{A}$ and $\mathbf{B}$ square symmetric (but not necessarily $\mathbf{a}$, because $\mathbf{B} \neq \mathbf{O})$. Condition (43) implies that also $R^{U}=0$, eqs. $\left(22_{4}\right)$ and $\left(25_{2}\right)$; moreover, we have already seen that whenever $\mathbf{B}$ is square symmetric, $\mathbf{V}$ is null, hence condition $\left(37_{2}\right)$ is satisfied. Also in this case it can be proved that condition $\left(37_{3}\right)$ is satisfied too, but once again, the analytical developments are too long to be presented here. So, it is sufficient to search for a stacking sequence that satisfies eqs. (43) and which is not uncoupled to obtain a coupled warp-free laminate.

The solutions proposed by Chen, [1] are all of this type:

$$
\begin{gathered}
\zeta_{1}+i \zeta_{3}=0, \quad \eta_{1}+i \eta_{3}=0, \\
{\left[\theta_{1},\left(\theta_{1}+\frac{\pi}{2}\right), \theta_{2},\left(\theta_{2}+\frac{\pi}{2}\right), \ldots \theta_{m},\left(\theta_{m}+\frac{\pi}{2}\right)\right] \text { such that } R_{1}^{B}=0,} \\
{\left[\left[\theta_{1},\left(\theta_{1}+\frac{\pi}{2}\right), \ldots, \theta_{m},\left(\theta_{m}+\frac{\pi}{2}\right)\right]_{\text {sym }}\right]_{-s y m},}
\end{gathered}
$$


where the subscript sym stand for symmetric and -sym for antisymmetric.

Conditions (44) are the most general case among those proposed by Chen: they simply correspond to eqs. (43), cfr. eqs. $\left(22_{4}\right)$ and $\left(23_{4}\right)$. The sequences in eq. (45) are sufficient to ensure $R_{1}^{A}=0$, while the angles of the sequences are searched for by a numerical technique in order to satisfy also $R_{1}^{B}=0$. The third case, sequences of the type (46), satisfies automatically eqs. (43), while $R_{0}^{B} \neq 0$, as it is easy to check using eqs. $\left(22_{4}\right),\left(23_{4}\right)$ and $\left(6_{1}\right)$. So, sequences (46) are sufficient to obtain a warp-free laminate, no matter of the values of the orientation angles $\theta_{j}$. Solutions proposed by Winckler, [19], are of the type

$$
\left[\theta,\left(\theta+\frac{\pi}{2}\right)_{2}, \theta,-\theta,-\left(\theta+\frac{\pi}{2}\right)_{2},-\theta\right],
$$

and it is evident that they are a particular case of the third one proposed by Chen, sequence (46). It can be also remarked, as already done in [2], that condition (44) implies also that $R^{U}=0$, eq. $\left(25_{2}\right)$, which means that the tensor of thermal expansion coefficients of the laminate, $\mathbf{U}$, is isotropic. So, solutions proposed by Winckler and Chen are warp-free laminates of the first type. Anyway, other solutions can exist, but they are to be searched for by a numerical optimization procedure.

5.2. Second type of warp-free laminates. The second type of warp-free coupled laminates is composed of all the laminates that satisfy

$$
\left\{\begin{array}{l}
\mathbf{B} \neq \mathbf{O} \\
\mathbf{V}=\mathbf{B A}^{-1} \mathbf{U}
\end{array}\right.
$$

Also conditions (48) are sufficient conditions to obtain a coupled laminate that satisfies eq. (18), i.e. a warp-free coupled plate. In this case too some remarks can be done.

Remark R8. Being $\mathbf{d} \neq \mathbf{O}$, conditions (37) and (48) are the only possible conditions giving a warp-free coupled laminate: no other types of warp-free coupled laminates can exist. Hence, a coupled laminate is warp-free if and only if it satisfies to one of the conditions (37) or (48). These are the necessary and sufficient conditions for a laminate to be warp-free, contrarily to what claimed in [2], where only solutions of the type (44) are considered, besides those having $\mathbf{B}=\mathbf{O}$, that are trivial and not considered in this paper because they are uncoupled.

Remark R9. Considering eqs. (48) it is clear that in this second case it is

$$
\mathbf{B} \neq \mathbf{O}, \quad \mathbf{V} \neq \mathbf{O}, \quad \mathbf{v}_{2}=\mathbf{O} .
$$

Remark R10. Comparing eqs. (49) with (41) shows that the set of the first type laminates can be considered, in some sense, as a subset of the second type laminates set. In fact, eq. (18), in the second type it is

$$
\mathbf{d} \mathbf{V}=-\mathbf{b}^{\mathrm{T}} \mathbf{U},
$$

while in the first type it is

$$
\mathbf{d} \mathbf{V}=-\mathbf{b}^{\mathrm{T}} \mathbf{U}=\mathbf{O},
$$


which is a particular case of the second one. Namely laminates composed of square symmetric layers belong to the first type, see remark $R 6$, because in such a case

$$
\mathbf{V}=\mathbf{B A}^{-1} \mathbf{U}=\mathbf{O} \text {. }
$$

Eq. (52) shows that in this peculiar case, in which $\mathbf{U}$ is isotropic, $\mathbf{U}$ is the eigentensor of tensor $\mathbf{B A}^{-1}$ relative to the null eigenvalue; this is possible because in this particular case, $\mathbf{B A}^{-1}$ is singular: using again Binet's theorem, one gets

$$
\operatorname{det}\left(\mathbf{B A}^{-1}\right)=\frac{\operatorname{det} \mathbf{B}}{\operatorname{det} \mathbf{A}}=0 \text {, }
$$

the last equality being ensured because $\operatorname{det} \mathbf{B}=0$, see remark $R 3$. The same happens when the layers are $R_{0}$-orthotropic, though in such a case $\mathbf{U}$ is no more isotropic.

Remark R11. Looking at eqs. (49) it is evident that in this second case, coupled warpfree laminates have nonetheless the thermo-elastic coupling stiffness tensor $\mathbf{V}$ which is not null. Actually, thermal stability is a condition depending essentially upon the compliance coupling tensors and it can exist also when stiffness coupling tensors are not null, as in this case. This, along with remark R1, confirms what announced in section 4: thermo-elastic coupling tensors can be null separately. Physically, eq. (49) implies that non mechanical moments, i.e. moments produced by thermal effects, can be nonzero, also without warping, which contradicts what said in [2]. This point is rather important, because it shows that the distinction made in this paper between first and second type of coupled warp-free laminates is not a merely taxonomic one, but really a physical one.

Remark R12. Eq. $\left(48_{2}\right)$ is a link between tensors that are not independent. It can be used to find some sufficient conditions for obtaining warp-free laminates of the second type. In fact, the polar components of the tensor $\mathbf{V}$ computed using the result provided by eq. $\left(48_{2}\right)$ are to be the same that can be computed using eqs. (26), in particular the isotropic part must be null. If $V_{11}, V_{22}$ and $V_{12}$ denote the Cartesian components of $\mathbf{V}$ computed as in $\left(48_{2}\right)$, the three conditions are

$$
\left\{\begin{array}{l}
V_{11}+V_{22}=0, \\
\frac{V_{11}-V_{22}}{2}+i V_{12}=\frac{h^{2}}{2} R^{\gamma} e^{2 i \Phi^{\gamma}}\left(\eta_{1}+i \eta_{3}\right) .
\end{array}\right.
$$

The above conditions can be directly given as relations among the polar parameters of $\mathbf{B}, \mathbf{A}$ and $\mathbf{U}$; the expressions are very long and cumbersome, so only they are omitted here. Nevertheless, it can be checked by some standard, though lengthy passages, that a sufficient condition for eq. (54) to be satisfied is that

$$
\frac{T^{\gamma}}{R^{\gamma}}=\frac{T_{1}}{R_{1}} .
$$

Eq. (55) is a relation on the polar parameters of $\mathbf{Q}$ and $\gamma$; hence, it regards the physical properties of the basic layer. This means that there is a class of warp-free laminates whose existence depends upon the physical properties of the material, not only upon the geometry of the stacking sequence. This circumstance confirms what has already been observed on other problems of optimal elastic properties of a laminate, [13]. 
The mathematical structure of eq. (55) is rather elegant: it is based upon the ratio of two homologous invariant quantities, one ratio concerning tensor $\gamma$, the other one tensor Q. Nevertheless, it is worth to rewrite eq. (55) with the Cartesian components of $\mathbf{Q}$ and $\gamma$; some simple manipulations give

$$
\left(Q_{y y y y}+Q_{x x y y}\right) \gamma_{x x}=\left(Q_{x x x x}+Q_{x x y y}\right) \gamma_{y y},
$$

which can still be transformed, using eq. (8), to let appear the components of the thermal expansion coefficients tensor, $\alpha$ :

$$
\left(Q_{x x x x} Q_{y y y y}-Q_{x x y y}^{2}\right)\left(\alpha_{y y}-\alpha_{x x}\right)=0 .
$$

This last condition shows that there are two possible cases: the first one is trivial, it is the case of isotropic thermal expansion coefficients, i.e. of $\alpha_{x x}=\alpha_{y y}$, a rare but possible case. The other one is given by the condition

$$
Q_{x x x x} Q_{y y y y}=Q_{x x y y}^{2},
$$

which written in terms of technical moduli reads like

$$
E_{x}=2 \nu_{x y}^{2} E_{y} .
$$

What is rather surprising, is that eqs. (55) and (56) concern both the thermal and elastic properties of the material, but when they are transformed into eq. (57), then one can immediately see that solutions can depend exclusively either on the thermal or the elastic properties of the material.

It is worth writing eq. (58) with the polar formalism; starting from eq. (29) written for the reduced stiffness tensor $\mathbf{Q}$, some simple passages lead to

$$
T_{1}^{L}\left[T_{0}^{L}+(-1)^{K^{L}} R_{0}^{L}\right]=2\left(R_{1}^{L}\right)^{2} .
$$

Condition (60) corresponds to a point on the boundary of the existence domain of the polar invariants for a planar material, see [10]. Hence, material dependent solutions satisfying condition (58) are only a limit case, while those satisfying $\alpha_{x x}=\alpha_{y y}$ are possible, though rare.

It is to be noticed that condition (55), and the other ones that follow from it, does not correspond to that given by Cross et al in [2] as a material dependent solution. In fact, it can be easily checked that the solution proposed by these authors corresponds to the case of a material with $R^{\gamma}=0$. This is a situation that always occurs when $R_{1}=0$, because a square symmetric layer, i.e. with $R_{1}=0$, has an isotropic tensor of thermal expansion coefficients, i.e. with $R^{\alpha}=0$, for obvious material symmetry reasons, and these two facts give always $R^{\gamma}=0$, as it can be easily checked. Hence, the materialdependent solution proposed in [2] is actually a solution of the type in eq. (42) which is, contrarily to what claimed in [2], not material dependent (because, unlike the cases given by eq. (55), it depends only upon the existence of a special type of orthotropy of the basic layer and not upon the numerical value of its mechanical properties), really possible and even the easiest case to be obtained.

To remark that when $R_{1}^{A}=0, \mathbf{V}$ is not null, but when $R_{1}^{B}=0$, then $\mathbf{V}=\mathbf{O}$ and eq. $\left(37_{3}\right)$ is satisfied too: the solution is of the first type. 


\section{EXPANSION-FREE COUPLED LAMinATES}

With the expression expansion-free laminate we will indicate in the following a laminate that does not contract or expand when only the thermal field $\nabla t$ is present. The objective is to obtain coupled laminates satisfying eq. $\left(21_{1}\right)$. Eq. (17) gives two different cases for having $\mathbf{v}_{1}=\mathbf{O}$ when $\mathbf{B} \neq \mathbf{O}$ :

$$
\left\{\begin{array}{l}
\mathbf{B} \neq \mathbf{O}, \\
\mathbf{V}=\mathbf{O}, \\
\mathbf{b W}=\mathbf{O} .
\end{array}\right.
$$

and

$$
\left\{\begin{array}{l}
\mathbf{B} \neq \mathbf{O}, \\
\mathbf{V}=\mathbf{B D}^{-1} \mathbf{W} .
\end{array}\right.
$$

Comparing conditions (61) and (62) respectively with (37) and (48), it is easy to recognize that the case of expansion-free laminates is a sort of dual case of the warp-free laminates one. In fact, it is sufficient to replace, in (37) and (48), $\mathbf{U}$ with $\mathbf{W}$ and $\mathbf{A}$ with $\mathbf{D}$ to obtain, apart the transpose of $\mathbf{b}$, the same conditions of (61) and (62).

This means that all the remarks done for warp-free laminates can be repeated almost verbatim for expansion-free laminates. Namely, it is possible to obtain laminates having $\mathbf{V}=\mathbf{O}$ and $\mathbf{v}_{1} \neq \mathbf{O}$, or with $\mathbf{v}_{1}=\mathbf{O}$ and $\mathbf{V} \neq \mathbf{O}$, still confirming what already said in section 4. In addition, eq. (42) are also sufficient conditions to obtain expansion-free laminates of the first type, i.e. satisfying conditions (61): laminates composed by identical layers reinforced by balanced fabrics are also expansion-free. Another sufficient condition for obtaining expansion-free laminates of the first type can be obtained by eq. (43) replacing tensor $\mathbf{A}$ with tensor $\mathbf{D}$ :

$$
R_{1}^{D}=R_{1}^{B}=0 .
$$

Conditions (54) are valid also in this case for the solutions of the second type, eq. (62): condition (55) is a sufficient condition also for obtaining material dependent expansionfree laminates; of course, the comments made in remark $R 12$ are valid also in this case.

To be remarked that sequences (44) to (47) do not have, generally speaking, $R_{1}^{D}=0$, so they are not expansion-free laminates.

\section{WARP-FREE AND EXPANSION-FREE COUPLED LAMINATES}

We ponder now if thermally stable laminates, i.e. laminates being at the same time warp-free and expansion-free, can exist. To be thermally stable, a laminate must satisfy both conditions (21). Considering hence conditions (37), (48), (61) and (62), one can see immediately that there are still two sets of solutions to this problem. 
7.1. First type of coupled warp-free and expansion-free laminates. The first one, is the set composed by the laminates that satisfy at the same time both conditions (37) and (61):

$$
\left\{\begin{array}{l}
\mathbf{B} \neq \mathbf{O} \\
\mathbf{V}=\mathbf{O} \\
\mathbf{b}^{\mathrm{T}} \mathbf{U}=\mathbf{O} \\
\mathbf{b W}=\mathbf{O}
\end{array}\right.
$$

It is self evident that conditions (42) are again sufficient conditions for having expansionfree and warp-free laminates. Another sufficient condition is obtained by the union of conditions (43) and (63):

$$
R_{1}^{A}=R_{1}^{B}=R_{1}^{D}=0 ;
$$

using eqs. (22) - (27), consequences of eqs. (65) are

$$
R^{U}=R^{V}=R^{W}=0,
$$

i.e. these laminates have a perfectly uncoupled isotropic thermal behaviour: the coefficients of thermal expansion or curvature do not depend upon the direction, they can be computed using eqs. (16) and (19) and their common value $\varepsilon$ is

$$
\varepsilon=t^{u}=t^{w}=\frac{T^{\gamma}}{4 T_{1}} .
$$

In the equation above, $t^{u}$ and $t^{w}$ indicate the isotropic polar component of tensors $\mathbf{u}$ and $\mathbf{w}$ respectively. To be remarked that such a value of $\epsilon$ cannot be modified, and by consequence it cannot be optimized by a proper choice of the layer orientations, because it depends only on the isotropic parts of $\gamma$ and $\mathbf{Q}$, quantities belonging to the basic layer. The value of $\varepsilon$ can be given as function of the polar components of only $\mathbf{Q}$ and $\alpha$ : using eq. (8) and indicating with $t, r$ and $\varphi$ the polar components of the tensor $\alpha$ of thermal expansion coefficients of the layer, one gets, after some standard manipulations,

$$
\varepsilon=t+(-1)^{k} r \frac{R_{1}}{T_{1}}
$$

which shows that this isotropic thermal expansion coefficient is nonetheless affected by the anisotropic coefficients $r$, of thermal expansion of the layer, and $R_{1}$. Exponent $k$ is an integer that can take two values:

$$
k= \begin{cases}0, & \text { if } \quad \varphi=\Phi_{1}, \quad \text { i.e. if } \quad \alpha_{11}>\alpha_{22}, \\ 1, & \text { if } \quad \varphi=\Phi_{1}+\frac{\pi}{2}, \quad \text { i.e. if } \quad \alpha_{11}<\alpha_{22} .\end{cases}
$$

7.2. Second type of warp-free and expansion-free laminates. The second set of warp-free and expansion-free laminates, is composed by the laminates that satisfy at the same time both conditions (48) and (62):

$$
\left\{\begin{array}{l}
\mathbf{B} \neq \mathbf{O}, \\
\mathbf{V}=\mathbf{B A}^{-1} \mathbf{U}, \\
\mathbf{V}=\mathbf{B D}^{-1} \mathbf{W} .
\end{array}\right.
$$


Solutions to eq. (70) are necessarily material dependent. Of course, conditions $\left(70_{2}\right)$ and $\left(70_{3}\right)$ give the following one, that can be obtained also through conditions (20) and $\left(48_{2}\right)$ :

$$
\mathbf{A}^{-1} \mathbf{U}=\mathbf{D}^{-1} \mathbf{W}
$$

It is easy to see that the last equation is valid also when written for the normalized tensors:

$$
\begin{array}{lll}
\mathbf{A}^{*}=\frac{\mathbf{A}}{h}, & \mathbf{B}^{*}=2 \frac{\mathbf{B}}{h^{2}}, & \mathbf{D}^{*}=12 \frac{\mathbf{D}}{h^{3}} \\
\mathbf{U}^{*}=\frac{\mathbf{U}}{h}, & \mathbf{V}^{*}=2 \frac{\mathbf{V}}{h^{2}}, & \mathbf{W}^{*}=12 \frac{\mathbf{W}}{h^{3}} .
\end{array}
$$

A particular set of laminates satisfies automatically condition (71). Introducing the homogeneity tensors $\mathbf{C}$ and $\mathbf{Z}$,

$$
\begin{aligned}
& \mathbf{C}=\mathbf{A}^{*}-\mathbf{D}^{*}, \\
& \mathbf{Z}=\mathbf{U}^{*}-\mathbf{W}^{*},
\end{aligned}
$$

one can see immediately that condition (71), when written using the normalized tensors (72), is identically satisfied whenever $\mathbf{C}$ is null. Actually, this is a sufficient condition to have also $\mathbf{Z}=\mathbf{O}$, as it is easily recognized writing the polar components of $\mathbf{C}$ and $\mathrm{Z}$ :

$$
\begin{aligned}
& \left\{\begin{array}{l}
T_{0}^{C}=0, \\
T_{1}^{C}=0, \\
R_{0}^{C} e^{4 i \Phi_{0}^{C}}=R_{0} e^{4 i \Phi_{0}} \sum_{j=1}^{n} c_{j} e^{4 i \delta_{j}} \\
R_{1}^{C} e^{2 i \Phi_{1}^{C}}=R_{1} e^{2 i \Phi_{1}} \sum_{j=1}^{n} c_{j} e^{2 i \delta_{j}} ;
\end{array}\right. \\
& \left\{\begin{array}{l}
T^{Z}=0 \\
R^{Z} e^{2 i \Phi^{Z}}=\frac{1}{n^{3}} R^{\gamma} e^{2 i \Phi^{\gamma}} \sum_{j=1}^{n} c_{j} e^{2 i \delta_{j}}
\end{array}\right.
\end{aligned}
$$

where

$$
c_{j}=n^{2}-d_{j}=4 n^{2}+6 n+4-12 j(j-n-1) .
$$

So, whenever laminates with $\mathbf{C}=\mathbf{O}$ are used, the second type of warp-free and expansionfree laminates can be found simply satisfying conditions $\left(70_{1}\right)$ and $\left(70_{2}\right)$ or $\left(70_{1}\right)$ and $\left(70_{3}\right)$. In particular, it is sufficient to use a laminate of the so-called quasi-trivial set of the type $\mathbf{C}=\mathbf{O}$. These laminates have been found by Vannucci and Verchery, [14], [15], and they ensure automatically conditions $\left(70_{1}\right)$ and (71) for any possible set of orientation angle of the layers. Using such quasi-trivial sequences, the only sufficient condition to be satisfied to obtain warp-free and expansion-free laminates of the second type is $\left(70_{2}\right)$, or alternatively $\left(70_{3}\right)$.

Finally, mixed cases, i.e. laminates satisfying conditions (37) and (62), or (48) and (61) cannot exist, because in both cases equation $\mathbf{V}=\mathbf{O}$ enter the set of conditions, forcing the solution to be one of the first type. 


\section{COMPletely WARP-FREE OR EXPANSION-FREE COUPLED LAMiNATES}

The case of coupled laminates is interesting, in principle, for another reason. In fact, let us consider again a laminate which is not acted upon by internal actions, i.e. $\mathbf{N}=\mathbf{M}=\mathbf{O}$. Then, eq. (11) gives that it is possible to obtain a laminate which is completely warp-free, for any possible distribution of the temperature, i.e. $\forall t$ and $\forall \nabla t$, when

$$
\left\{\begin{array}{l}
\mathbf{v}_{2}=\mathbf{O} \\
\mathbf{w}=\mathbf{O}
\end{array}\right.
$$

Condition $\left(77_{2}\right)$ is never satisfied for uncoupled laminates, because in such a case $\mathbf{v}_{2}$ and $\mathbf{b}$ are null and $\mathbf{w}$ reduces to, cfr. (19),

$$
\mathbf{w}=\mathbf{d W},
$$

which is never null. Unlike this case, when a laminate is coupled, w is null when, see again (19),

$$
\mathbf{V}=\mathbf{A B}^{-1} \mathbf{W}
$$

If this condition is added to (48), then the laminate will have

$$
\mathbf{B} \neq \mathbf{O}, \quad \mathbf{V} \neq \mathbf{O}, \quad \mathbf{b} \neq \mathbf{O}, \quad \mathbf{v}_{2}=\mathbf{O}, \quad \mathbf{w}=\mathbf{O},
$$

i.e. it will never warp for a thermal field.

In a similar way, it is also possible to find laminates that are expansion-free for any possible thermal field, when

$$
\left\{\begin{array}{l}
\mathbf{v}_{1}=\mathbf{O} \\
\mathbf{u}=\mathbf{O}
\end{array}\right.
$$

As before, condition $\left(81_{2}\right)$ is never satisfied by an uncoupled laminate, for which, see $(16)$,

$$
\mathbf{u}=\mathbf{a U},
$$

which is never null. Nevertheless, if the laminate is coupled, $\mathbf{u}$ is null if, once more cfr. (16),

$$
\mathbf{V}=\mathbf{D B}^{-1} \mathbf{U}
$$

condition that added to $(62)$ gives

$$
\mathbf{B} \neq \mathbf{O}, \quad \mathbf{V} \neq \mathbf{O}, \quad \mathbf{b} \neq \mathbf{O}, \quad \mathbf{v}_{\mathbf{1}}=\mathbf{O}, \quad \mathbf{u}=\mathbf{O} .
$$

These conditions show that such a laminate will never have a deformation of the midplane produced by a thermal field. To remark that $\mathbf{A}^{-1} \neq \mathbf{a}$ and $\mathbf{D}^{-1} \neq \mathbf{d}$ because $\mathbf{B} \neq \mathbf{O}$.

Finally, if conditions (77) and (81) are imposed at the same time, then such a laminate will never freely deform or warp for the only action of a thermal field. These four 
conditions are not independent: using eqs. $\left(48_{2}\right),\left(62_{2}\right),(79)$ and $(82)$ gives the following three independent conditions:

$$
\left\{\begin{array}{l}
\mathbf{V}=\mathbf{B A}^{-1} \mathbf{U} \\
\mathbf{W}=\left(\mathbf{B A}^{-1}\right)^{2} \mathbf{U} \\
\mathbf{D}=\mathbf{B A}^{-1} \mathbf{B}
\end{array}\right.
$$

This results implies that it is not possible to have 3 tensors simultaneously null among $\mathbf{u}, \mathbf{v}_{1}, \mathbf{v}_{2}$ and $\mathbf{w}$ : the only possible situations are one, two or all the four tensors null. Being conditions $\left(48_{2}\right)$ and $\left(62_{2}\right)$ dependent upon the material, solutions to eq. (84) are material dependent too.

Using the same above conditions, it can be proved, by the polar method, that laminates with $\mathbf{C}$ null cannot satisfy at the same time conditions (77) and (81).

\section{Final Remarks AND PERSPECtives}

The general theory of thermally-stable laminates has been presented in this paper. The conditions giving all the possible types of warp-free or/and expansion-free laminates have been presented, their link with special orthotropies of the basic layer or of the stiffness tensors of the laminate has been discussed. The existence of special solutions, depending on the physical properties of the material, has been demonstrated too. Also the existence, in principle, of coupled laminates that are completely insensitive to thermal fields has been predicted.

In other papers, some of the results presented in this one have been found by an approach based upon the use of Cartesian components and some special, but partial, considerations. Unlike in such cases, the results found in this paper have been obtained by a direct tensor approach and thanks to the polar formalism. The use of such a tensor representation has proved to be very effective, not only for this research, especially because it is based upon the use of tensor invariants that are directly linked to some elastic symmetry properties. This fact has been fundamental in the present study: for instance, it has been helpful in determining different results, and namely in analyzing the effects of the special orthotropies $R_{0}=0$ and $R_{1}=0$.

The distinction made in the paper between solutions of the first and second type is crucial, not only upon the mathematical point of view, but also for the mechanical aspects: the laminates belonging to the two types have actually different mechanical responses. Another interesting point, at least under the theoretical point of view, is the fact that the true necessary and sufficient conditions for a laminate to be warp-free or expansion-free have been correctly given in the paper. Several other results never clearly exposed before are contained in the remarks presented in this paper.

Coupled thermally-stable laminates can be used in several applications, and in such cases normally designers want to optimize a certain parameter, for instance a Cartesian component of the coupling tensors, $\mathbf{B}$ or $\mathbf{b}$. In such cases, the conditions of thermalstability found in the paper become a constraint to an optimum design problem, where the objective is the Cartesian component to be optimized. Problems of this type have 
been already solved numerically in the past by the author and co-workers, using the polar formalism and metaheuristics, like for instance genetic algorithms or particle-swarm optimization, e.g. [11], [16], [18]. The research is going on in this direction for solving some problems of interest in practical applications of thermally stable laminates.

\section{REFERENCES}

[1] Chen, H.P.: Study on hygrothermal isotropic layup and hygrothermal curvature-stable coupling composite laminates. In: Proc. 44th AIAA/ASME/ASCE/AHS/ASC Structures, Structural Dynamics and Materials Conf., Paper No. AIAA-2003-1506. (2003)

[2] Cross, R.J., Haynes, R.A., Armanios, E.A.: Families of hygrothermally stable asymmetric laminated composites. Journal of Composite Materials 42, 697-716 (2008)

[3] Hahn, H.T.: Warping of unsymmetric cross-ply graphite/epoxy laminates. Composites Technology Review 3, 114-117 (1981)

[4] Haynes, R.A., Armanios, E.A.: New families of hygrothermally stable composite laminates with optimal extension-twist coupling. AIAA Journal 48, 2954-2961 (2010)

[5] Hyer, M.W.: Calculations of room-temperature shapes of unsymmetric laminates. Journal of Composite Materials 15, 296-310 (1981)

[6] Hyer, M.W.: Some observations on the cured shape of unsymmetric laminates. Journal of Composite Materials 15, 175-194 (1981)

[7] Jones, R.M.: Mechanics of composite materials. McGraw-Hill (1975)

[8] Tsai, S.W., Hahn, T.: Introduction to composite materials. Technomic (1980)

[9] Vannucci, P.: A special planar orthotropic material. Journal of Elasticity 67, 81-96 (2002)

[10] Vannucci, P.: Plane anisotropy by the polar method. Meccanica 40, 437-454 (2005)

[11] Vannucci, P.: Ale-pso : an adaptive swarm algorithm to solve design problems of laminates. Algorithms 2, 710-734 (2009)

[12] Vannucci, P.: Influence of invariant material parameters on the flexural optimal design of thin anisotropic laminates. International Journal of Mechanical Sciences 51, 192-203 (2009)

[13] Vannucci, P.: Strange laminates. Mathematical Methods in the Applied Sciences (in press), DOI: 10.1002/mma.2539 (2012)

[14] Vannucci, P., Verchery, G.: A special class of uncoupled and quasi-homogeneous laminates. Composites Science and Technology 61, 1465-1473 (2001)

[15] Vannucci, P., Verchery, G.: Stiffness design of laminates using the polar method. International Journal of Solids and Structures 38, 9281-9294 (2001)

[16] Vannucci, P., Vincenti, A.: The design of laminates with given thermal/hygral ex- pansion coefficients: a general approach based upon the polar-genetic method. Composite Structures 79, 454-466 (2007)

[17] Verchery, G.: Les invariants des tenseurs d'ordre $4 \mathrm{du}$ type de l'élasticité. In: Proc. of Colloque Euromech 115. Editions du CNRS, Villard-de-Lans (1979)

[18] Vincenti, A., Ahmadian, M.R., Vannucci, P.: Bianca: a genetic algorithm to solve hard combinatorial optimisation problems in engineering. Journal of Global Optimization 48, 399-421 (2010)

[19] Winckler, S.J.: Hygrothermally curvature stable laminates with tension-torsion coupling. Journal of the American Helicopter Society 31, 56-58 (1985)

[20] Winckler, S.J.: A theory of warp-free unsymmetric composite plates and beams with extensionbending coupling. Ph.D. thesis, Rensselaer Polytechnique Institute, Troy, NY (1986) 